\title{
Automated Noise-Parameter Measurements of Cryogenic LNAs
}

\author{
Alexander Sheldon ${ }^{*}$, Leonid Belostotski*, Hamdi Mani” ${ }^{*}$, Christopher E. Groppi ${ }^{\#}$, Karl F. Warnick ${ }^{\$}$ \\ *University of Calgary, Canada \\ \#Arizona State University, USA \\ \$Brigham Young University, USA \\ ${ }^{1}$ awsheldo@ucalgary.ca
}

\begin{abstract}
This paper addresses the need for measured cryogenic noise parameters. The measurement process is discussed and an analysis of the measurement uncertainty is performed. To verify proper operation of the measurement system, measurements of a 1-to-2-GHz radio-astronomy low-noise amplifier (LNA) at 20, 75, and $296 \mathrm{~K}$ are presented. In these measurements, the typical $1 \sigma$ measurement uncertainty in noise temperatures and minimum noise temperatures is $<10 \%$.

Keywords - Cryogenic electronics, low-noise amplifiers, noise parameters, radio astronomy, quantum computing.
\end{abstract}

\section{INTRODUCTION}

In many emerging fields, cryogenic low-noise amplifiers (cryo-LNAs) are required, and researchers can benefit from more sophisticated cryogenic measurement techniques. For decades, radio-astronomy telescopes have used cryo-LNAs to improve their sensitivity [1]. While conventional telescopes use a single receiver per antenna, the current trend is to employ antenna arrays for increased survey speeds [2]. Cryo-LNAs are also required for telescope receivers employing microwave kinetic inductance detectors [3], [4]. Quantum computers drastically increased interest in cryo-LNAs to reduce the thermal load in dilution cryostats due to interconnects to the room-temperature electronics by moving room-temperature electronics closer to qubits [5], [6]. In all cases, to achieve optimum noise performance from cryo-LNAs, the ability to measure their noise parameters is vital. The noise parameters include the minimum noise temperature, $T_{\min }$, the noise resistance, $R_{n}$, and the optimum reflection coefficient or admittance, $\Gamma_{\text {opt }}$ or $Y_{\text {opt }}$.

To measure the noise parameters, the output noise power spectra of the LNA are measured for at least four different source impedances [7]-[9]. At room temperature, these impedances are commonly generated with mechanical impedance tuners; however, such tuners are much too large to fit in cryostats. Placing them outside restricts measurements due to unknown noise contribution of a cryogenic-to-room-temperature interconnect, whose loss also restricts the attainable impedances. Prior to this work, two techniques to measure noise parameters at cryogenic temperatures either required cooling of large devices [10] or neglecting the error due to the unknown noise of the interconnect [11], [12]. A noise model for the interconnect was proposed in [13], but the performance cannot be verified without measurement of its temperature profile. This work presents the first automated method of measuring cryogenic noise parameters. The method is demonstrated with a radio-astronomy cryo-LNA.

Radio-astronomy telescope sensitivity is maximized to observe deeper into space and further back in time. The sensitivity is proportional to $A_{e f f} / T_{\text {sys }}$, where $A_{e f f}$ is the telescope effective collecting area and $T_{\text {sys }}$ is the system noise temperature. As there are physical and budgetary constraints to increasing $A_{e f f}$, effort is concentrated into decreasing $T_{\text {sys }}$ for higher sensitivity. The system noise temperature, $T_{s y s}$, is limited by the front-end LNA noise temperature, $T_{L N A}$. To minimize $T_{L N A}$, cryogenic cooling is commonly used [1]. Although methods of reducing $T_{L N A}$ guided by LNA noise parameters are well known, they are difficult to implement at cryogenic temperatures due to the lack of experimental validation. Commonly, cryo-LNAs are designed using 'rules of thumb' and approximations potentially producing sub-optimum designs. For optimum designs, the noise parameters of the devices at cryogenic temperatures and various biases are required.

This paper discusses a noise-parameter measurement process in Section II, with measurement results of a 1-to-2-GHz radio-astronomy cryo-LNA for the ALPACA array [2] presented in Section II-D. An analysis of the measurement uncertainty of both the noise-temperature and noise-parameter measurements are presented in Section III.

\section{THEORY OF OPERATION}

Noise-parameter measurements rely on measurements of noise temperatures (or noise figures). There are two methods of measuring cryogenic noise temperatures: the cold-source method [14] and the cold-attenuator method [15]. The cold-attenuator method is more common as it permits simultaneous measurement of the device-under-test (DUT) gain; however, it requires an external-to-cryostat calibrated noise source, which can potentially cause significant measurement errors due to temperature gradients and errors in the noise-source calibration. When measuring cryo-LNAs with sub-10-K noise temperatures, these errors can lead to significant measurement uncertainty, even when reduced by the cold attenuator. The cold-source method requires accurate measurements of the DUT S-parameters and the temperature of a cryogenically cooled $50 \Omega$ termination. Despite these additional measurements, the cold-source method eliminates errors due to the unknown noise temperature of the input cryogenic-to-room-temperature 


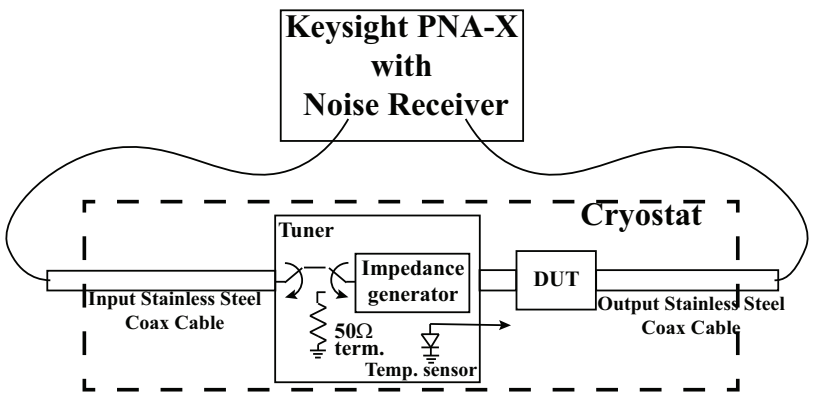

Fig. 1. Block diagram of the measurement system.



Fig. 2. Photo of the tuner and DUT inside the cryostat.

interconnect. Additionally, this method reduces measurement time by eliminating a noise-power-spectra measurement.

The DUT noise temperatures are measured when terminated by each of four signal-source impedances generated by a modified version of a NoiseTech C-IG0160C solid-state impedance generator (also known as an impedance tuner) placed in the cryostat at the input of the DUT. With just four operating states, this tuner realizes impedances that conform to impedance regions in [9] over its operating frequency range, thereby guaranteeing a diagonal-dominant linear system of equations. Furthermore, this tuner uses a switch to select either a thru state for S-parameter measurements or a built-in $50 \Omega$ termination for the cold-source noise-parameter measurement. This switch reduces the number of cooling cycles. An on-board temperature sensor measures the $50 \Omega$ termination temperature.

A block diagram of the measurement system is in Fig. 1. Both the S-parameters of the tuner+DUT and the output noise power spectra are measured by a Keysight PNA-X. A photo of the inside of the measurement cryostat is in Fig. 2.

\section{A. Calibration}

Prior to DUT measurements, calibration is required to determine S-parameters of the tuner, $S_{\text {tuner }}$, and the stainless-steel coaxial cables, and the noise temperature and gain of the noise receiver. These are required for each frequency point and operating temperature.

The S-parameters of the coaxial cable, $S_{\text {coax }}$, are determined by connecting a "SHORT" calibration standard inside the cryostat to each cable, measuring the resultant reflection coefficient, and fitting a transmission-line model.
While this approach is less accurate than, for example, a TRL method, it results in negligible degradation in accuracy as discussed in Section III and it reduces the number of cooling cycles to two: one each to measure the S-parameters of the tuner and the "SHORT" standard. The resultant $S_{\text {coax }}$ is then de-embedded from the S-parameters of the tuner+coax measured by the VNA to obtain $S_{\text {tuner }}$. For the cryostat used in this work, each of the two cooling cycles takes $\sim 8 \mathrm{~h}$ to cool to the operating temperature, $\sim 2 \mathrm{~h}$ to change temperature, if required, and $\sim 2 \mathrm{~h}$ to warm up. These calibration results are repeatable, due to components being relatively stationary, and the calibration can be reused for multiple measurements.

The noise temperature and the transducer gain of the noise receiver are obtained with a standard Y-factor measurement by connecting a calibrated noise source to the receiver input and collecting power spectra, $N_{\text {hot }}$ and $N_{\text {cold }}$, with the noise source in the ON and OFF states, respectively. From $N_{\text {hot }}$ and $N_{\text {cold }}$, the receiver gain, $G_{P N A}$, and noise temperature, $T_{P N A}$, are calculated as

$$
\begin{gathered}
G_{P N A}=\frac{N_{h o t}-N_{\text {cold }}}{T_{0} \times E N R} \\
T_{P N A}=\frac{N_{\text {cold }}}{G_{P N A}}-T_{\text {cold }}
\end{gathered}
$$

where $T_{0}$ is the IEEE noise reference temperature $(290 \mathrm{~K})$, the excess noise ratio (ENR) is $E N R=\left(T_{\text {hot }}-T_{\text {cold }}\right) / T_{0}$, and $T_{\text {hot }}$ and $T_{\text {cold }}$ are the noise-source temperatures in the $\mathrm{ON}$ and OFF states. For improved accuracy, the noise parameters of the receiver could be measured and the impedance mismatch between the noise source and the receiver could be accounted for as in [8]. However, due to the large LNA gain and very low reflection coefficient of the noise source and the noise receiver, these extra measurements were not implemented.

\section{B. Measurement}

At each cryogenic temperature, measurement steps include:

1) Measure the physical temperature of the termination, $T_{\text {term }}$, with the tuner temperature sensor at each desired temperature of the cryostat;

2) Measure the $S$-parameters of the tuner+DUT cascade, $S_{\text {tuner }+D U T}$, in the tuner THRU state;

3) Measure the output noise power spectra, $N_{i}: i=$ $A, B, C, D$, with the receiver while cycling through the four tuner impedance states (identified as A, B, C, and D) with the cold termination is switched in.

The measurement process is completely automated by a computer running MATLAB. The DUT measurements are completed in one cooling cycle, for any number of temperatures/biases, and take $\sim 10 \mathrm{~h}$ per temperature/bias point in large part due to 2901 frequency points, 256 averages for each impedance state, and the receiver bandwidth of $0.8 \mathrm{MHz}$. Increasing the receiver bandwidth would decrease measurement time significantly but may introduce measurement errors due to long coaxial cables [16]. Measuring at fewer frequency points can also speed up measurements until the cooling cycle dominates. 


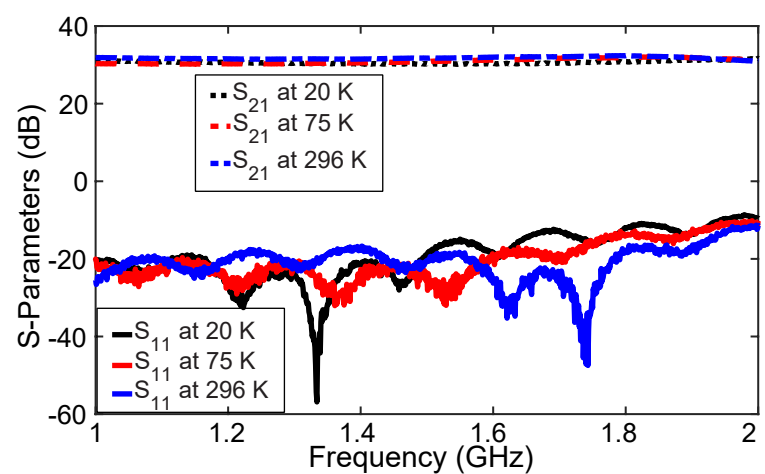

Fig. 3. S-parameters of the ALPACA LNA at $20 \mathrm{~K}, 75 \mathrm{~K}$, and $296 \mathrm{~K}$.

\section{Post-Processing}

S-parameters of the DUT, $S_{D U T}$, are obtained by de-embedding $S_{\text {tuner }}$ and $S_{\text {coax }}$ from $S_{\text {tuner }+D U T}$. The available gain, $G_{A, i}$, of the DUT cascaded with the output coax is calculated for each of the four source impedances, $Z_{s, i}=Y_{s, i}^{-1}$, presented by the tuner to the DUT.

For each of the tuner states, the noise temperature, $T_{i}$, is

$$
T_{i}=\frac{N_{i}}{G_{P N A} G_{A, i}}-\frac{T_{P N A}}{G_{A, i}}-T_{\text {term }},
$$

which is related to noise parameters by

$$
T_{i}=T_{\text {min }}+T_{0} \frac{R_{n}}{\Re\left(Y_{s, i}\right)}\left|Y_{s, i}-Y_{o p t}\right|^{2},
$$

from which the noise parameters, $T_{m i n}, R_{n}$, and $Y_{o p t}$ are found [7], [9].

\section{Measurement of ALPACA LNA}

To verify the operation of the measurement system, a 1-to-2-GHz radio-astronomy cryo-LNA, designed for the ALPACA array [2], was measured. For the 20-K measurement the cryostat was set to $15 \mathrm{~K}$, but due to the thermal load of the DUT, its temperature was measured to be $20 \mathrm{~K}$. The measured S-parameters are shown in Fig. 3, and the measured noise parameters are shown in Fig. 4 with $2 \sigma$ error bars that are discussed in Section III. The measurements verify near-optimum performance; however, in the $20-\mathrm{K}$ measurements a $\sim 1 \mathrm{~K}$ improvement could be made in the low-frequency noise temperature.

\section{Measurement-UnCertainty Analysis}

Both the calibration and the measurements lead to uncertainty in the final results. To estimate the uncertainty, a sensitivity analysis of (3) determines each contributor to the overall uncertainty in the noise temperature, given the measurement equipment uncertainty from datasheets as reported in Table 1.

All sources of uncertainty in Table 1 are assumed uncorrelated, and the total uncertainty is the sum-of-squares of the individual uncertainties. The uncertainties in $T_{i}$ were found by an analytic sensitivity analysis, where measurements in Section II-D served as the expected values. A summary
Table 1. Equipment measurement uncertainty.

\begin{tabular}{l|l|l|l}
\hline Measurement & Uncertainty & Distribution & Notes \\
\hline $\begin{array}{l}\text { Temperature } \\
\left(T_{\text {term }}\right)\end{array}$ & $0.33 \mathrm{~K}$ & Gaussian & $\begin{array}{l}\text { estimated } \\
\text { with } \\
\text { measurements }\end{array}$ \\
\hline $\begin{array}{l}\text { Noise Source } \\
\text { ENR }\end{array}$ & $0.05 \mathrm{~dB}$ & Gaussian & $\begin{array}{l}\text { Keysight } \\
\text { N4002A }\end{array}$ \\
\hline $\begin{array}{l}\text { Noise Power } \\
\left(N_{i}, N_{\text {hot }},\right.\end{array}$ & $0.003 \mathrm{~dB}$ & Gaussian & $\begin{array}{l}\text { Keysight } \\
\text { N5242A, } \\
\left.N_{\text {cold }}\right)\end{array}$ \\
$\begin{array}{l}\text { S-parameters } \\
\left(S_{\text {tuner }+D U T},\right.\end{array}$ & $\begin{array}{l}\text { mean=-55dB, } \\
\text { max=-50dB }\end{array}$ & Rayleigh & $\begin{array}{l}\text { Keysight } \\
\text { N5242A }[8]\end{array}$ \\
$\begin{array}{l}\left.S_{\text {tuner }}\right) \\
\text { Coax Model } \\
\left(S_{\text {coax }}\right)\end{array}$ & $0.033 \mathrm{~dB} \mathrm{\&}$ & Gaussian & $\begin{array}{l}\text { max. error in } \\
\text { model fit }\end{array}$ \\
\hline
\end{tabular}

Table 2. Contributions to the measurement uncertainty of $T_{i}$ in (3).

\begin{tabular}{l|l|l|l|l}
\hline & \multicolumn{4}{|c}{ Uncertainty in $T_{i}(\mathrm{~K})$} \\
\hline Source & State A & State B & State C & State D \\
\hline$T_{\text {term }}$ & 0.7 & 0.7 & 0.7 & 0.7 \\
\hline$T_{P N A}$ & 0.13 & 0.56 & 0.21 & 0.21 \\
\hline$G_{P N A}$ & $\sim 0$ & $\sim 0$ & $\sim 0$ & $\sim 0$ \\
\hline$G_{A, i}$ & 1.3 & 2.6 & 2.6 & 1.1 \\
\hline$N_{i}$ & $\sim 0$ & $\sim 0$ & $\sim 0$ & $\sim 0$ \\
\hline Total & $\mathbf{1 . 5}$ & $\mathbf{2 . 8}$ & $\mathbf{2 . 7}$ & $\mathbf{1 . 3}$ \\
\hline \multicolumn{5}{|l}{}
\end{tabular}

of the maximum $1 \sigma$ uncertainty contributions to the total uncertainty in noise temperatures are shown in Table 2. Since only the uncertainties due to $T_{\text {term }}$ and $G_{A, i}$ contribute significantly to the total uncertainty, the resultant analytic sensitivity expression can be simplified as

$$
\Delta T_{i} \approx \sqrt{\Delta T_{\text {term }}^{2}+4 T_{i}^{2}\left(1-10^{\Delta S_{21, d B} / 20}\right)^{2}} .
$$

Equation (5) shows that $\Delta T_{i}$ is dominated by the uncertainty in physical temperature of the termination and S-parameter measurements and can guide future improvements to the measurement process.

Uncertainties in $T_{i}$ affect the accuracy in noise-parameter determination from (4). Since the system of equations originating from (4), does not have a closed-form solution for the noise parameters, further analytic analysis of the uncertainty is difficult. Instead, following [8], [17], MATLAB is used to complete a Monte Carlo analysis. The measured temperatures, S-parameters, and noise powers are perturbed according to Table 1. The Monte Carlo simulation is run for 1000 iterations and uncertainties of the noise parameters are determined and shown with $2 \sigma$ error bars in Fig. 4. Although not shown in the figures, these error bars reduce significantly with averaging of repeated measurements. For noise temperatures and minimum noise temperatures, the typical $1 \sigma$ uncertainty is $<10 \%$.

\section{CONCLUSION}

This work demonstrates the first fully automated measurement of cryogenic noise parameters. This is possible because of the impedance tuner located inside the cryostat. 

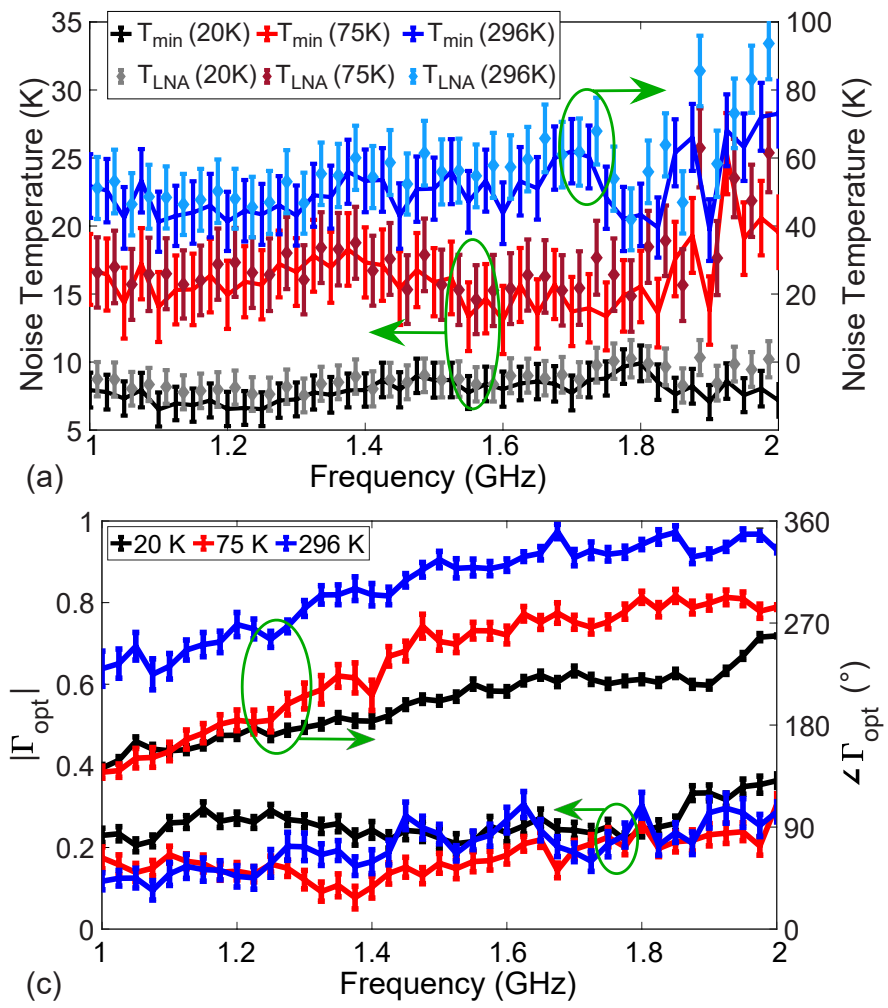
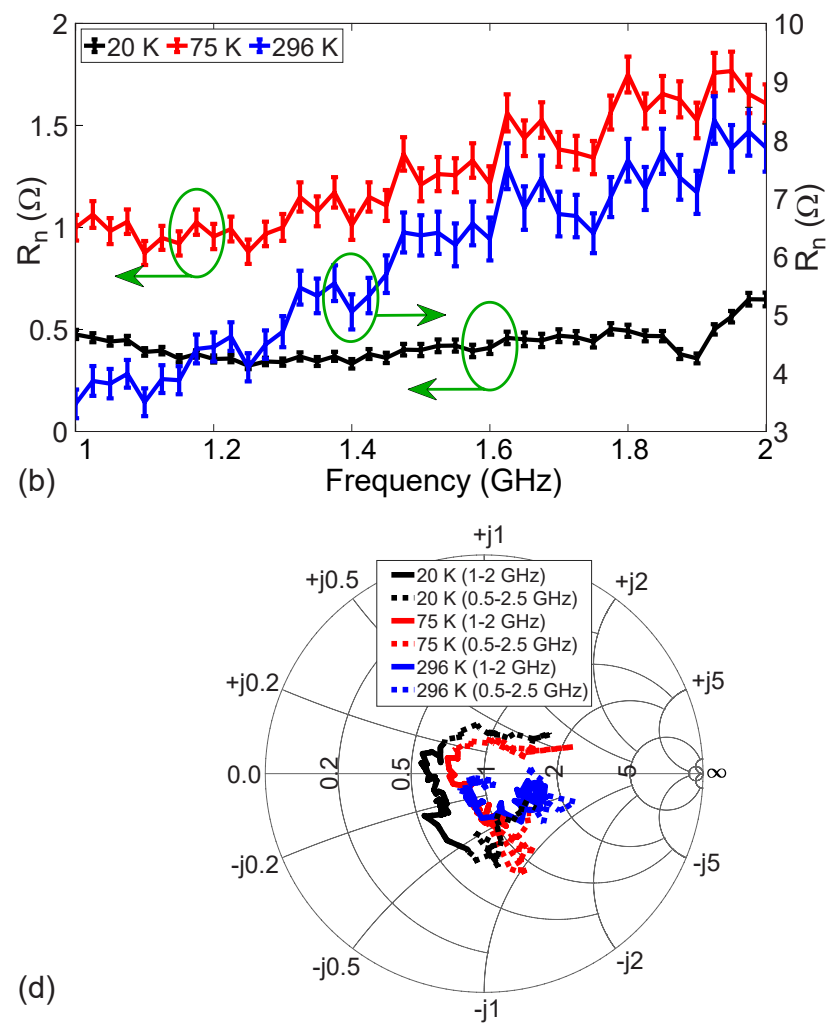

Fig. 4. Measured (a) $T_{\min }$ and $T_{L N A}$, (b) $R_{n}$, and (c),(d) $\Gamma_{o p t}$ of the ALPACA LNA at $20 \mathrm{~K}, 75 \mathrm{~K}$, and $296 \mathrm{~K}$ with $2 \sigma$ error bars.

This automation permits in situ measurements at different physical temperatures and different biases, which enables characterization and optimization of cryo-LNAs. Measured noise parameters reveal how well the LNA is noise matched and the best noise temperature achievable by the LNA. The measured noise parameters have $1 \sigma$ measurement uncertainties of $<10 \%$ for $T_{L N A}$ and $T_{\min },<4 \%$ for $R_{n},<12 \%$ for $\left|\Gamma_{\text {opt }}\right|$, and $<8^{\circ}$ for $\angle \Gamma_{\text {opt }}$.

\section{ACKNOWLEDGMENT}

This work was funded in part by the University of Calgary, NSERC (Grants: RGPIN/03855-2018, RGPAS/522621-2018), Canada Research Chair program (950-228619, 950-231990), CFI (Grant: 228619), Alberta Economic Development and Trade (Grant: RCP-14-022-SEG), and CMC Microsystems. This material is also based upon work supported by the U.S. NSF under Grant No. 1636645.

\section{REFERENCES}

[1] J. Schleeh et al., "Cryogenic LNAs for SKA band 2 to 5," in IEEE Int. Microwave Symp., Honolulu, June 2017, pp. 164-167.

[2] G. Cortes-Medellin et al., "A fully cryogenic phased array camera for radio astronomy," IEEE Trans. Antennas Propag., vol. 63, no. 6, pp. 2471-2481, June 2015.

[3] P. K. Day et al., "A broadband superconducting detector suitable for use in large arrays," Nature, vol. 425, no. 6960, p. 817, 2003.

[4] M. Hosseini, W. Wong, and J. C. Bardin, "A 0.4-1.2 GHz SiGe Cryogenic LNA for Readout of MKID Arrays," in IEEE Int. Microwave Symp., Boston, June 2019, pp. 164-167.

[5] B. Patra et al., "A scalable cryo-CMOS 2-to-20GHz digitally intensive controller for $4 \times 32$ frequency multiplexed spin qubits/transmons in 22nm FinFET technology for quantum computers," in IEEE Int. Solid-State Circ. Conf., San Francisco, Feb. 2020, pp. 304-306.
[6] L. Le Guevel et al., "A $110 \mathrm{mK} 295 \mu \mathrm{W} 28 \mathrm{~nm}$ FDSOI CMOS quantum integrated circuit with a $2.8 \mathrm{GHz}$ excitation and nA current sensing of an on-chip double quantum dot," in IEEE Int. Solid-State Circ. Conf., San Francisco, Feb. 2020, pp. 306-307.

[7] R. Q. Lane, "The determination of device noise parameters," Proceedings of the IEEE, vol. 57, no. 8, pp. 1461-1462, Aug 1969.

[8] L. Belostotski and J. W. Haslett, "Evaluation of tuner-based noise-parameter extraction methods for very low noise amplifiers," IEEE Trans. Microw. Theory Techn., vol. 58, no. 1, pp. 236-250, Jan. 2010.

[9] M. Himmelfarb and L. Belostotski, "On impedance-pattern selection for noise parameter measurement," IEEE Trans. Microw. Theory Techn., vol. 64, no. 1, pp. 258-270, Jan. 2016.

[10] R. Hu and S. Weinreb, "A novel wide-band noise-parameter measurement method and its cryogenic application," IEEE Trans. Microw. Theory Techn., vol. 52, no. 5, pp. 1498-1507, May 2004.

[11] I. Rolfes, T. Musch, and B. Schiek, "Cryogenic noise parameter measurements of microwave devices," IEEE Trans. Instrum. Meas., vol. 50, no. 2, pp. 373-376, Apr. 2001.

[12] W. Wiatr, "Comments on "cryogenic noise parameter measurements of microwave devices"," p. 619, Apr. 2004.

[13] S. Colangeli et al., "Evaluation of coaxial cable performance under thermal gradients," Int. J. Microwave and Wireless Tech., vol. 7, no. 3-4, pp. 239-249, 2015.

[14] J. Randa et al., "Precision measurement method for cryogenic amplifier noise temperatures below $5 \mathrm{~K}$," IEEE Trans. Microw. Theory Techn., vol. 54, no. 3, pp. 1180-1189, March 2006.

[15] J. E. Fernandez, "A noise-temperature measurement system using a cryogenic attenuator," Telecommunications and Mission Operations Progress Report, vol. 135, pp. 1-9, Jul 1998.

[16] A. Sheldon et al., "Impact of noise bandwidth on noise figure," IEEE Trans. Instrum. Meas., vol. 68, no. 7, pp. 2662-2664, July 2019.

[17] J. Randa, "Noise-parameter uncertainties: A Monte Carlo simulation," Journal of Research of the National Institute of Standards and Technology, vol. 107, no. 5, pp. 431-444, Sept. 2002. 